\title{
K ATP Permanent Neonatal Diabetes
}

National Cancer Institute

\section{Source}

National Cancer Institute. KATP Permanent Neonatal Diabetes. NCI Thesaurus. Code C131848.

K ATP channel-associated neonatal diabetes mellitus that does not resolve spontaneously. 